\title{
Smoking status and anti-inflammatory macrophages in bronchoalveolar lavage and induced sputum in COPD
}

Lisette IZ Kunz ${ }^{1 *}$, Thérèse S Lapperre ${ }^{1}$, Jiska B Snoeck-Stroband ${ }^{2}$, Simona E Budulac ${ }^{3}$, Wim Timens ${ }^{4}$, Simone van Wijngaarden ${ }^{1}$, Jasmijn A Schrumpf ${ }^{1}$, Klaus F Rabe ${ }^{1}$, Dirkje S Postma ${ }^{5}$, Peter J Sterk ${ }^{1,6}$ and Pieter $\mathbf{S}$ Hiemstra ${ }^{1}$ and Groningen Leiden Universities Corticosteroids in Obstructive Lung Disease (GLUCOLD) study group'P.S.Hiemstra@lumc.nl

\begin{abstract}
Background: Macrophages have been implicated in the pathogenesis of COPD. M1 and M2 macrophages constitute subpopulations displaying pro- and anti-inflammatory properties. We hypothesized that smoking cessation affects macrophage heterogeneity in the lung of patients with COPD. Our aim was to study macrophage heterogeneity using the M2-marker CD163 and selected pro- and anti-inflammatory mediators in bronchoalveolar lavage (BAL) fluid and induced sputum from current smokers and ex-smokers with COPD.

Methods: 114 COPD patients (72 current smokers; 42 ex-smokers, median smoking cessation 3.5 years) were studied cross-sectionally and underwent sputum induction (M/F 99/15, age $62 \pm 8$ [mean \pm SD] years, 42 (31-55) [median (range)] packyears, post-bronchodilator FEV $63 \pm 9 \%$ predicted, no steroids past 6 months). BAL was collected from 71 patients. CD163 $3^{+}$macrophages were quantified in BAL and sputum cytospins. Pro- and antiinflammatory mediators were measured in BAL and sputum supernatants.
\end{abstract}

Results: Ex-smokers with COPD had a higher percentage, but lower number of CD163 ${ }^{+}$macrophages in BAL than current smokers (83.5\% and 68.0\%, $p=0.04 ; 5.6$ and $20.1 \times 10^{4} / \mathrm{ml}, \mathrm{p}=0.001$ respectively). The percentage CD163 M2 macrophages was higher in BAL compared to sputum $(74.0 \%$ and $30.3 \%, p<0.001)$. BAL M-CSF levels were higher in smokers than ex-smokers $(571 \mathrm{pg} / \mathrm{ml}$ and $150 \mathrm{pg} / \mathrm{ml}, \mathrm{p}=0.001)$ and correlated with the number of CD $163^{+}$BAL macrophages (Rs $=0.38, p=0.003$ ). No significant differences were found between smokers and exsmokers in the levels of pro-inflammatory (IL-6 and IL-8), and anti-inflammatory (elafin, and Secretory Leukocyte Protease Inhibitor [SLPI]) mediators in BAL and sputum.

Conclusions: Our data suggest that smoking cessation partially changes the macrophage polarization in vivo in the periphery of the lung towards an anti-inflammatory phenotype, which is not accompanied by a decrease in inflammatory parameters.

\section{Background}

Chronic obstructive pulmonary disease (COPD) is characterized by progressive lung function decline and an abnormal inflammatory response in the airways, mainly caused by cigarette smoke [1]. The inflammation response in the small airway in COPD is characterized

\footnotetext{
* Correspondence: L.I.Z.Kunz@lumc.nl

'Department of Pulmonology, Leiden University Medical Center, Leiden, The Netherlands

Full list of author information is available at the end of the article
}

by the accumulation of macrophages, neutrophils, CD8 ${ }^{+}$-lymphocytes and B-cells and is associated with the severity of COPD [2,3]. Smoking cessation is an effective treatment to reduce lung function decline [1]. Nevertheless, airway inflammation in bronchial biopsies, sputum and bronchoalveolar lavage (BAL) of COPD patients (predominantly) persists one year after smoking cessation [4-6]. We previously showed that the number of macrophages and neutrophils in bronchial biopsies are comparable in current and ex-smokers with COPD [7].
C Biomed Central

(c) 2011 Kunz et al; licensee BioMed Central Ltd. This is an Open Access article distributed under the terms of the Creative Commons Attribution License (http://creativecommons.org/licenses/by/2.0), which permits unrestricted use, distribution, and reproduction in any medium, provided the original work is properly cited. 
However, the effects of smoking on macrophage phenotypes in COPD are incompletely understood.

Macrophages play an important role in innate and adaptive immunity and form a heterogeneous population $[8,9]$. Macrophages display polarized phenotypes by which they can be divided into subpopulations. Proinflammatory, or classically activated macrophages (M1) display pro-inflammatory and cytotoxic properties and can eradicate intracellular pathogens. In contrast, antiinflammatory or alternatively activated macrophages (M2) display anti-inflammatory properties and are implicated in repair $[8,10]$. Granulocyte-macrophage colony stimulating factor (GM-CSF) can generate M1 in vitro from human peripheral blood monocytes, and macrophage colony stimulating factor (M-CSF) can generate M2 [11]. M1 secrete pro-inflammatory cytokines, like IL-(Interleukin)-12 and tumor necrosis factor (TNF)- $\alpha$, have good antigen presenting capacity and promote Th1 immunity. In contrast, M2 secrete anti-inflammatory mediators, such as IL-10, show poor antigen presenting capacity and promote development of T-regulatory cells [11-13]. Alveolar macrophages show anti-inflammatory M2-characteristics [14-16], which can be distinguished from pro-inflammatory macrophages using M2 markers such as the scavenger receptor CD163 [17,18]. Compared to M1 cells, M2 macrophages are highly phagocytic. The phagocytic capacity of alveolar macrophages is decreased in smoking COPD patients and improves with smoking cessation [19]. This suggests a phenotypic alteration and a role of macrophage heterogeneity in COPD, which has also been proposed in e.g. tumor progression [20], atherosclerosis [21] and renal diseases [22].

Although inflammation persists, smoking cessation shows positive clinical effects [1]. This suggests that other mechanisms play a beneficial role, for instance regulation of macrophage polarization. We hypothesize that in moderate to severe COPD patients $i$ ) ex-smokers have more M2 and anti-inflammatory mediators in BAL and induced sputum compared to current smokers; ii) M2 and anti-inflammatory mediators are relatively higher in the peripheral airways (as sampled by BAL) than in the central airways (as sampled by induced sputum).

\section{Methods}

\section{Subjects and study design}

Patient characteristics and methods have been described previously $[7,23,24]$. In short, we studied 114 clinically stable moderate to severe COPD patients [GLUCOLD study (Groningen Leiden Universities Corticosteroids in Obstructive Lung Disease)] cross-sectionally. They were aged 45-75 years, smoked $\geq 10$ packyears and were current or ex-smokers (quit $\geq 1$ month). Patients diagnosed with asthma, $\alpha 1$-antitrypsin deficiency and those who used corticosteroids in the past six months were excluded; they were allowed to use short-acting bronchodilators. Approval of the medical ethics committees of both centers was obtained and all patients provided written informed consent [23]. Spirometry was performed according to international guidelines [25]. All patients underwent a bronchoscopy with BAL and a sputum induction on separate visits.

\section{Bronchoscopy, BAL and sputum induction}

Fiberoptic bronchoscopy was performed in all patients and processed using a standardized protocol, as previously described $[7,24,26,27]$. The BAL procedure was discontinued during the study due to ethical considerations, since four of 71 patients experienced a serious adverse event that was considered to be possibly related to the BAL procedure (pleural pain, fever, pneumonia, short-term cardiac ischemia). Sputum induction was achieved using hypertonic sodium chloride aerosols $(\mathrm{w} / \mathrm{v}$ 4.5\%) for a maximal duration of three times five minutes and processed according to the whole sample method.

\section{BAL and sputum processing}

BAL was filtered through a nylon gauze and centrifuged for 10 minutes at $450^{*} \mathrm{~g}$ at $4^{\circ} \mathrm{C}$. If erythrocytes were macroscopically present, the cell pellet was resuspended in lysisbuffer (100 $\mathrm{ml}$ phosphate buffered saline (PBS) containing 0.83 gram $\mathrm{NH}_{4} \mathrm{Cl}, 0.1$ gram $\mathrm{KHCO}_{3}$ and 0.004 gram Ethylenediaminetetra Acetic Acid (EDTA), $\mathrm{pH} 7.4)$ for 5 minutes and centrifuged $\left(450^{*} \mathrm{~g}, 4^{\circ} \mathrm{C}\right)$. The cell pellet was resuspended in $0.1 \%$ glucose $(w / v)$ in PBS and centrifuged again under the same conditions. BAL processing and differential cell counts were performed analogous to the methods described for sputum processing, except that no dithiothreitol was used for homogenization. The viability of the non-squamous cells in BAL was similar in smokers and ex-smokers (82 $\pm 12 \%$ versus $82 \pm 9 \%, \mathrm{p}=0.96)$.

Sputum was processed according to the whole sample method and all samples were treated with dithiothreitol 0.1\% (DTT, Sputolysin, Calbiochem) [28]. Cell free supernatants of both BAL and sputum were stored at $-80^{\circ} \mathrm{C}$.

From both BAL and sputum samples cytospins were centrifuged on apex-coated slides [28]. A sputum sample was considered adequate when the percentage squamous cells was less than $80 \%$. After drying for 1 hour, the cytospins were wrapped in aluminum foil and stored at $-80^{\circ} \mathrm{C}$ pending immunocytochemical staining.

\section{Immunocytochemical staining}

Frozen cytospins of BAL and sputum were brought to room temperature in one hour. BAL cytospins were 
fixed in acetone at $-20^{\circ} \mathrm{C}$ for 10 minutes, dried and endogeneous peroxidase activity was blocked by incubation in methanol and $0.3 \%$ hydrogen peroxide for 10 minutes. Sputum cytospins were fixed in $4 \%$ paraformaldehyde in PBS $0.9 \%(\mathrm{w} / \mathrm{v})$ for 1 hour, rinsed with PBS and endogenous peroxidase activity was blocked with sodium azide $0.1 \%(\mathrm{w} / \mathrm{v})$ and hydrogen peroxide $0.18 \%$ $(w / v)$ in PBS for 30 minutes. Non-specific binding was blocked in PBS, $1 \%$ bovine serum albumin (BSA) and 5\% normal human serum (NHS) for 45 minutes for the sputum cytospins only. Mouse-anti-human CD163 (clone GHI/61, BD Pharmingen) was used as a primary antibody to stain M2-type macrophages [17] at the dilution of 1:75 for BAL cytospins and 1:50 for sputum cytospins, and both were incubated for one hour at room temperature. The primary antibody was diluted in $\mathrm{PBS} / 1 \%$ BSA for BAL cytospins and in PBS/1\%BSA/1\% NHS for sputum cytospins. The horseradish peroxidase conjugated anti-mouse Envision system (DAKO, Glostrup, Denmark) was used as a secondary antibody and was incubated for 30 minutes, the chromogen NovaRed (Vector, Burlingame, CA) for 7 minutes. All washing steps were with PBS. All slides were counterstained with Mayer's hematoxylin (Klinipath, Duiven, The Netherlands) and mounted afterwards with Pertex mounting medium (HistoLab, Gothenburg, Sweden).

We considered the possibility that DTT used to liquefy the induced sputum samples affects detection of CD163. To this end we generated M1 and M2 by culture of monocytes for six days in the presence of GMCSF and M-CSF respectively [11], and treated these cells with DTT prior to FACS-based analysis of CD163 expression and preparation of cytospins followed by immunocytochemical staining for CD163.

\section{Analysis of cytospins}

Two cytospins per sample were stained for differential cell counts with May-Grünwald Giemsa (MGG). Differential cell counts were expressed as a percentage of nucleated cells, squamous cells excluded. The median percentage squamous cells was $7.5 \%$ (2.1-13.3\%). CD163 ${ }^{+}$ and $\mathrm{CD}_{163}{ }^{-}$macrophages were enumerated based on morphology by two independent, experienced researchers at $400 \times$ magnification (figure 1). To avoid observer bias, slides were coded without knowledge of clinical data. The mean number of $\mathrm{CD} 163^{+}$macrophages divided by the total counted number of macrophages was used to calculate the percentage of $\mathrm{CD} 163^{+}$ macrophages. The total number of $\mathrm{CD}_{163^{+}}$macrophages per volume was calculated by the percentage of $\mathrm{CD} 163^{+}$macrophages multiplied by the total number of macrophages. Repeatability between the two observers (LIK and SVW) was good, as measured by the intraclass coefficient (ICC), with the two way random model and absolute agreement. For BAL $\mathrm{CD} 163^{+}$and CD163- macrophages the ICC were both 95\%; for sputum $\mathrm{CD}_{163^{+}}$and $\mathrm{CD} 163^{-}$macrophages the ICC were 97\% and $93 \%$ respectively.

\section{Enzyme-linked Immunosorbent Assay (ELISA)}

Commercially available kits were used to detect GMCSF (Bender Medsystems), M-CSF (R\&D systems), IL-6, IL-8, IL-10 (Sanquin), IL-12 (IL-12/IL-23p40, R\&D systems) and elafin (HBT) in sputum and BAL supernatants. SLPI ELISA was developed in our laboratory at the Leiden University Medical Center [29]. The absorbance was measured at $450 \mathrm{~nm}$ using a Microplate reader (model 680; Bio-Rad, Hercules, CA) and Microplate Manager software (version 5.2.1, Bio-Rad). The lower limits of detection for sputum were $300 \mathrm{pg} / \mathrm{ml}$ (SLPI), $2.5 \mathrm{ng} / \mathrm{ml}$ (elafin), $38 \mathrm{pg} / \mathrm{ml}$ (IL-6) and $400 \mathrm{pg} /$ $\mathrm{ml}$ (IL-8). The lower limits of detection for BAL were $150 \mathrm{pg} / \mathrm{ml}$ (M-CSF), $0.2 \mathrm{ng} / \mathrm{ml}$ (SLPI), $5.5 \mathrm{pg} / \mathrm{ml}$ (IL-6) and $15 \mathrm{pg} / \mathrm{ml}$ (IL-8). In BAL and sputum supernatants, IL-10, IL-12, GM-CSF levels were below the lower limit of detection. Furthermore, elafin and M-CSF were undetectable in BAL and sputum supernatants respectively. In case more than $10 \%$ of the samples were below the detection limits, the value of these samples was set at the lower limit of detection (M-CSF and IL-6 in BAL).

\section{Statistical analysis}

Mean values and standard deviations (SD) or medians with interquartile ranges (IQR) are presented. When appropriate, variables were logarithmically transformed before statistical analysis. Differences between smokers and ex-smokers were explored using $\chi^{2}$-tests, two-tailed unpaired t-tests and Mann-Whitney tests. We used the Spearman (Rs) correlation coefficient to analyze correlations. Multiple linear regression was used to correct for the recovery of BAL. The statistical analysis was performed with SPSS 16.0 software (SPSS Inc., Chicago, IL). Statistical significance was inferred at $\mathrm{p}<0.05$.

\section{Results}

\section{Characteristics}

In total, 114 COPD patients participated in the study, 72 current smokers and 42 ex-smokers, as presented in table 1. All steroid-naive patients had moderate to severe COPD (GOLD stage II-III) based on a mean (SD) post-bronchodilator $\mathrm{FEV}_{1}$ of 63 (9)\% predicted and had a median $\left(25^{\text {th }}\right.$ and $75^{\text {th }}$ percentile) smoking history of 42 (31-55) packyears. The total group of patients and the unselected group in which BAL was performed were comparable. Of the BAL samples (first 71 patients), 62 were suitable for analysis. 106 out of 109 sputum inductions were suitable for analysis. BAL and sputum cell differentials and cell concentrations are presented in 


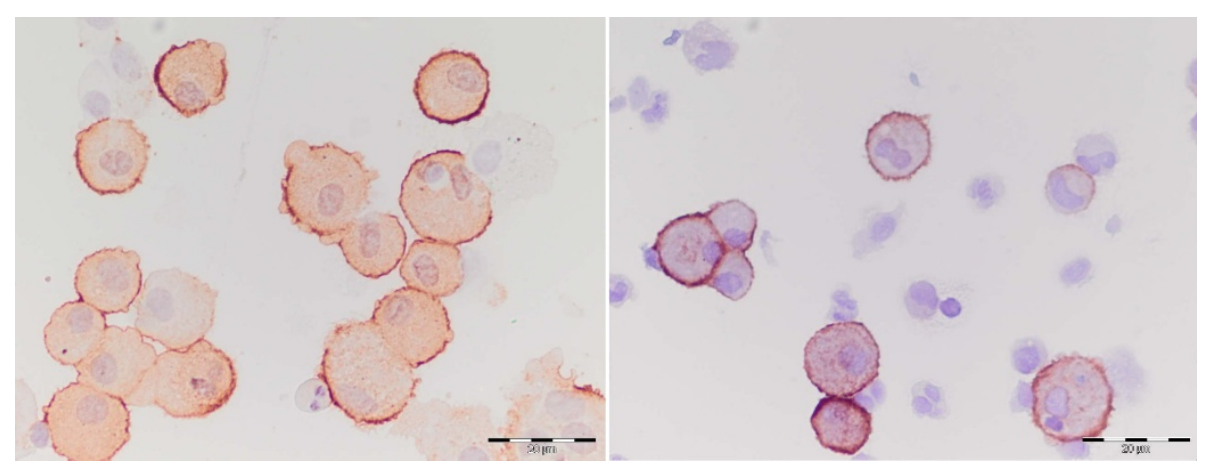

Figure 1 Photomicrograph of membrane-bound CD163 staining on BAL and sputum cells. A BAL cytospin is shown in the left photograph and a sputum cytospin in the right photograph. Scale bar $=20 \mu \mathrm{m}$.

figures 2 and 3. The percentage and number of macrophages in BAL were significantly higher in current smokers than in ex-smokers $(95.8 \%$ and $74.2 \%, \mathrm{p}<0.001$; 34.0 and $7.6 \times 10^{4} / \mathrm{ml}, \mathrm{p}=0.008$ respectively). The mean recovery of BAL was $41(18) \%$; the recovery in smokers was higher compared to ex-smokers (45 (16)\% and $35(19) \%, \mathrm{p}=0.039$ respectively).

\section{Smoking status and $\mathrm{CD} 163^{+}$macrophages in BAL and induced sputum}

DTT used to liquefy the induced sputum did not affect detection of CD163 by FACS and immunocytochemical staining (data not shown). Ex-smokers with COPD had a significantly higher percentage of anti-inflammatory $\mathrm{CD}_{163^{+}}$macrophages in BAL than current smokers (83.5\% and $68.0 \%, \mathrm{p}=0.04$, respectively) (figure 4), independent of BAL recovery. However, ex-smokers had a lower number of anti-inflammatory macrophages in BAL compared to current smokers (5.6 and $20.1 \times 10^{4}$ / $\mathrm{ml}, \mathrm{p}=0.001$, respectively). The percentage $\mathrm{CD} 163^{+}$

Table 1 Patient characteristics for current and ex-smokers with COPD

\begin{tabular}{lll}
\hline & $\begin{array}{l}\text { Smokers } \\
(\mathbf{n}=\mathbf{7 2})\end{array}$ & $\begin{array}{l}\text { Ex-smokers } \\
(\mathbf{n}=\mathbf{4 2})\end{array}$ \\
\hline Males (n (\%)) & $59(81.9)$ & $40(95.2)^{*}$ \\
Age (years) & $60.1(7.7)$ & $64.1(7.2)^{*}$ \\
Packyears & $43.3(32.4-55.6)$ & $36.8(27.5-53.1)$ \\
Smoking cessation (years) & & $3.5(1.0-9.8)$ \\
FEV 1 post-bronchodilator (L) & $2.02(0.46)$ & $2.05(0.46)$ \\
FEV $_{1}$ post-bronchodilator (\%pred) & $63.3(8.3)$ & $62.5(9.6)$ \\
FEV $_{1}$ /IVC\% post-bronchodilator & $49.5(8.5)$ & $46.0(8.3)^{*}$ \\
$\mathrm{~K}_{\mathrm{co}}$ (\%pred) & $73.3(25.1)$ & $80.4(25.9)$ \\
\hline
\end{tabular}

Data are presented as mean (SD) or median (IQR) unless otherwise stated. These patient characteristics have been previously described [7]. pred $=$ predicted; $\mathrm{FEV}_{1}=$ forced expiratory volume in one second; IVC = inspiratory vital capacity; $\mathrm{K}_{\mathrm{CO}}=$ carbon monoxide transfer coefficient. * $\mathrm{p}<0.05$ compared with smokers with COPD $\left(\chi^{2}\right.$ test for sex differences, two tailed unpaired t-tests for other data). macrophages was higher in BAL compared to sputum (74.0\% and 30.3\%, p < 0.001, respectively). Ex-smokers had a similar percentage and number of anti-inflammatory macrophages in induced sputum compared to current smokers with COPD $(25.0 \%$ and $31.1 \%, \mathrm{p}=0.89$; 10.1 and $6.8 \times 10^{4} / \mathrm{ml}, \mathrm{p}=0.24$ respectively).

\section{Smoking status and soluble mediators in BAL and induced sputum supernatants}

BAL M-CSF levels were lower in ex-smokers than current smokers $(\mathrm{p}=0.001)$ (figure 5 and table 2$)$. This difference was neither explained by differences in BAL recovery between both groups, nor by the ratio of $\mathrm{M}$ CSF to anti-inflammatory macrophages. No correlation was found between recovery and BAL M-CSF levels. The anti-inflammatory mediator SLPI in BAL was inversely correlated with recovery. The pro-inflammatory mediators IL- 6 and IL-8 in BAL were comparable between smokers and ex-smokers and were independent of recovery. No difference was found in induced sputum for the pro-inflammatory IL-6, IL-8 levels and the antiinflammatory mediator elafin. The levels of SLPI, IL-6 and IL-8 in sputum were higher than the levels in BAL (all p < 0.001). M-CSF was below the lower limits of detection in induced sputum and elafin was undetectable in BAL.

\section{Correlation between cells, mediators and lung function}

The number of $\mathrm{CD}_{163^{+}}$macrophages in BAL correlated with $\mathrm{FEV}_{1}$ post-bronchodilator (\%predicted) $(\mathrm{Rs}=0.255$; $\mathrm{p}=0.05)$ and $\mathrm{FEV}_{1} / \mathrm{IVC} \%(\mathrm{Rs}=0.374 ; \mathrm{p}=0.004)$. No correlations were found between the number and percentage $\mathrm{CD}_{163^{+}}$macrophages in BAL and sputum and the number of packyears or the duration of smoking cessation. No correlations were found between the number of packyears or duration of smoking cessation and concentrations of all soluble mediators in BAL and induced sputum. 

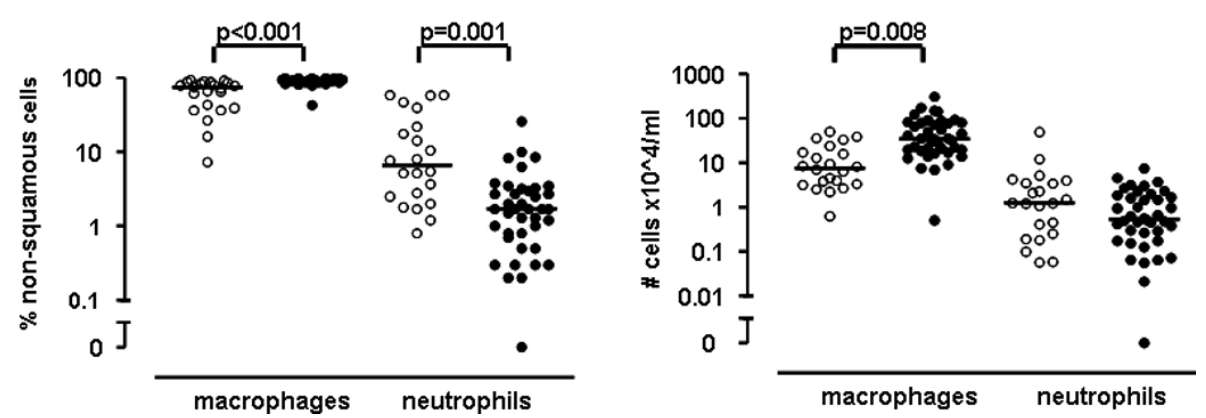

Figure 2 BAL differential cell counts expressed as percentage and cell concentrations of COPD patients. Percentage is shown in the left panel, cell concentrations in the right panel. Open circles represent ex-smokers, closed circles represent current smokers. Horizontal bars represent medians. P-values are corrected for recovery of BAL fluid using multiple linear regression.

BAL M-CSF correlated with the number of CD163 macrophages in BAL ( $\mathrm{Rs}=0.379 ; \mathrm{p}=0.003)$. BAL SLPI was negatively correlated with the number and percentage of macrophages and positively correlated with the number and percentage of neutrophils in BAL (all p < $0.05)$. BAL SLPI and the number of $\mathrm{CD}_{163}{ }^{+}$macrophages correlated inversely $(\mathrm{Rs}=-0.353 ; \mathrm{p}=0.008)$. Sputum SLPI correlated with the number and percentage of $\mathrm{CD} 163^{+}$macrophages in sputum ( $\mathrm{Rs}=0.377$; $\mathrm{p}<0.001$ and $\mathrm{Rs}=0.236 ; \mathrm{p}=0.021$, respectively). Both BAL and sputum IL-8 correlated inversely with percentage macrophages, but positively with the percentage and number of neutrophils (all p $<0.05$ ). This relation was not seen for IL-6. A trend was seen for a correlation between sputum IL- 8 and the percentage of CD163 ${ }^{+}$ macrophages $(\mathrm{Rs}=-0.189 ; \mathrm{p}=0.061)$. The percentage, but not the number, of $\mathrm{CD}_{163^{+}}$macrophages in BAL showed a trend for correlation with sputum ( $\mathrm{Rs}=0.267$, $\mathrm{p}=0.053)$.

\section{Discussion}

This study is the first to show that the percentage of macrophages with anti-inflammatory, M2-type characteristics (as shown by CD163 expression) is significantly higher in BAL from ex-smokers than in current smokers with COPD. In addition, the percentage of anti-inflammatory macrophages was higher in BAL than in induced sputum, indicating a predominance of this macrophage phenotype in the periphery of the lung. BAL M-CSF correlated with the number of CD163 ${ }^{+}$ macrophages in BAL. The results together are in line with the hypothesis that smoking cessation causes a shift in the phenotype of luminal macrophages towards a more anti-inflammatory phenotype, which is restricted to the periphery of the lung. Although we did observe a higher percentage of M2-type macrophages in BAL from ex-smokers, this was not accompanied by a decrease in inflammatory parameters such as neutrophils and pro-inflammatory mediators.

Our study shows that ex-smokers with COPD have a higher percentage of anti-inflammatory macrophages in BAL than current smokers. Our findings on pulmonary macrophage polarization further extend previous observations. First, we discovered that macrophages recovered from induced sputum have less anti-inflammatory features than from BAL. A previous study showed that induced sputum of COPD patients contains a majority of pro-inflammatory macrophages, based on their

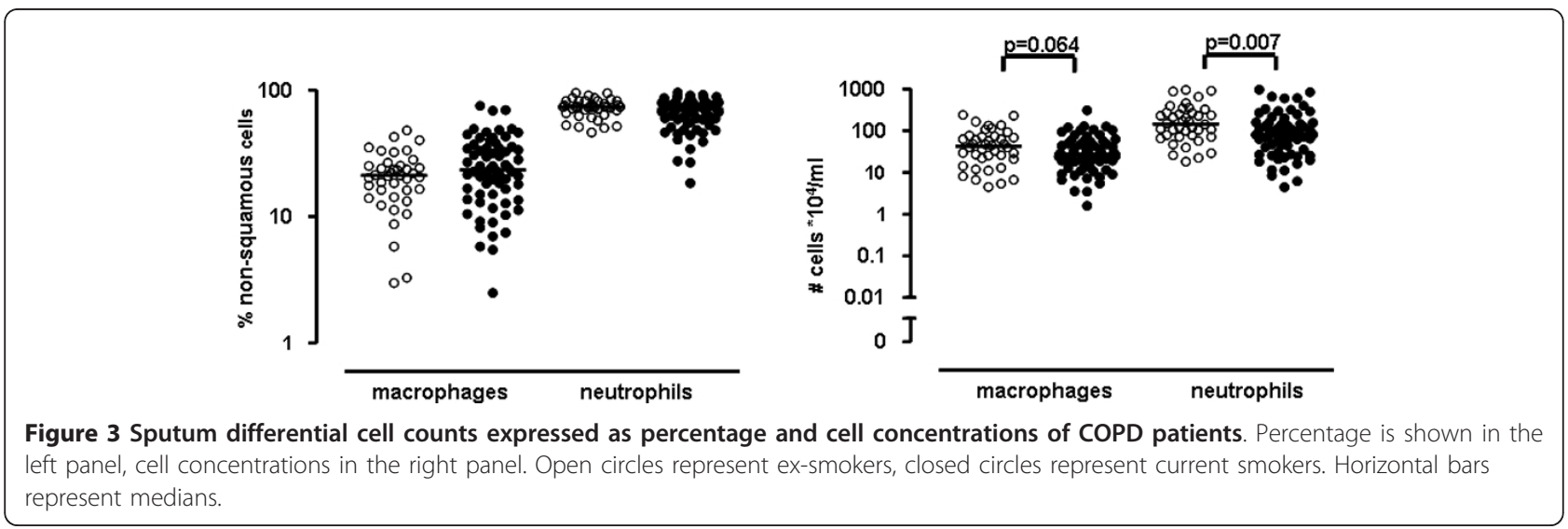



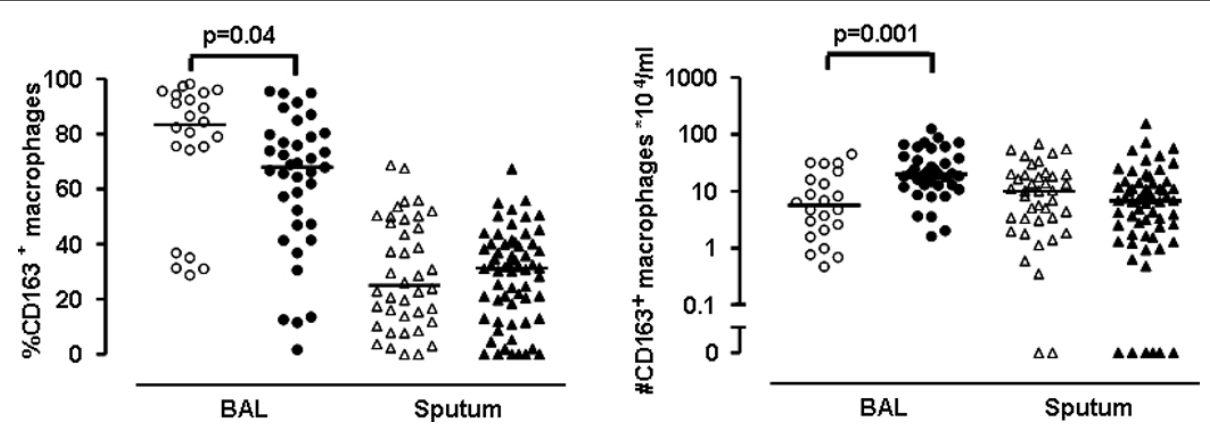

Figure 4 The percentage and number of $\mathrm{CD} 163^{+}$macrophages in BAL and induced sputum in COPD patients. The percentage (left panel) and number of $\mathrm{CD}_{163^{+}}$macrophages (right panel) in BAL and induced sputum between ex-smokers (open symbols) and smokers (closed symbols) with COPD. Horizontal bars represent medians. P-values are corrected for recovery of BAL fluid using multiple linear regression.

HLA-DR expression and capacity to produce TNFa, in contrast to control subjects [30]. However, these authors only analyzed markers of pro-inflammatory macrophages and most patients used corticosteroids which may have affected the macrophage phenotype [17]. Second, we showed that ex-smokers have more antiinflammatory macrophages in BAL than current smokers. This is in line with a recent paper, showing that never smokers compared to current smokers had higher BAL levels of CCL18, a chemokine expressed by alternatively activated macrophages [31]. Furthermore, previous studies have shown that anti-inflammatory macrophages have a higher phagocytic capacity $[8,10]$. Therefore our findings are in line with another study demonstrating that alveolar macrophages of current smokers with COPD show reduced phagocytosis compared to exsmokers [19]. In addition, active smoking, but also the presence of COPD itself, may be associated with an impaired phagocytic capacity of alveolar macrophages (and therefore a predominance of pro-inflammatory macrophages) [32-34]. However, in contrast to these and our findings, a recent study indicated that smoking may enhance macrophage differentiation into an antiinflammatory phenotype, since cigarette smoking polarized human alveolar macrophages of COPD patients in vivo towards an enhanced expression of M2-related genes and a suppression of M1 genes [35]. This study included only 12 COPD patients with predominantly GOLD stage I. A possible explanation for this apparent difference with our observations is therefore that the direction of the effect of smoking on macrophage differentiation may be determined by disease severity.

Previously, several studies have evaluated the effect of smoking on soluble mediators. We found comparable SLPI levels in BAL between current smokers and ex-smokers with COPD, in line with results from a

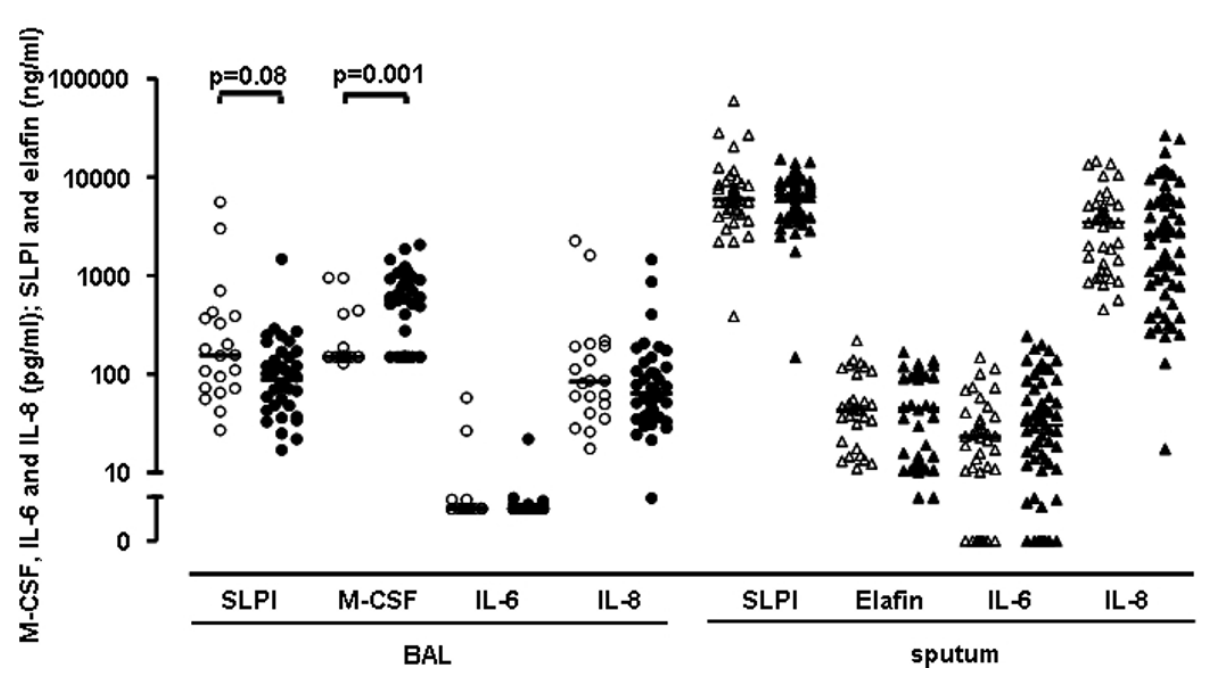

Figure 5 Soluble mediators measured in BAL and induced sputum supernatants of COPD patients. Ex-smokers are represented by open symbols and smokers by closed symbols. Horizontal bars represent medians. P-values are corrected for recovery of BAL fluid using multiple linear regression. 
Table 2 Soluble mediators measured in BAL and induced sputum supernatants of smokers and ex-smokers with COPD

\begin{tabular}{lll}
\hline Soluble mediator & Ex-smokers & Smokers \\
\hline BAL & & \\
SLPI (ng/ml) & $156(72-386)$ & $87(48-154)$ \\
M-CSF (pg/ml) & $150(150-159)$ & $571(150-927)$ \\
IL-6 (pg/ml) & $6(6-6)$ & $6(6-6)$ \\
IL-8 (pg/ml) & $83(43-193)$ & $64(37-122)$ \\
Sputum & & \\
SLPI (ng/ml) & $5897(4406-8628)$ & $6643(4321-8862)$ \\
Elafin (ng/ml) & $44(20-102)$ & $44(12-101)$ \\
IL-6 (pg/ml) & $23(10-36)$ & $30(11-58)$ \\
IL-8 (pg/ml) & $3454(1178-5212)$ & $2571(805-5900)$ \\
\hline
\end{tabular}

Data are presented as medians (IQR $25-75^{\text {th }}$ percentile).

study of 25 smoking, ex-smoking and never smoking COPD patients with GOLD stage II-III [36]. We did not find a difference in BAL IL- 6 and IL-8 and sputum IL-6 between current smokers and ex-smokers with COPD, in line with two previous studies $[37,38]$.

We believe that our study has several strengths. We studied a large cohort of well-characterized COPD patients in which sputum $(\mathrm{n}=114)$ and BAL $(\mathrm{n}=71)$ were collected, whereas previous studies were of smaller size $[30,31,36,39]$. In addition, we studied steroid-naive patients, excluding possible influences of inhaled corticosteroid therapy on CD163 expression. This is important, since it has been shown in previous studies that dexamethasone induces CD163 expression on monocytes and macrophages in vitro [17]. The BAL and sputum cytospins were counted manually by two independent researchers simultaneously (LIK and SVW). $\mathrm{CD}_{163}{ }^{-}$macrophages as well as $\mathrm{CD} 163^{+}$macrophages were readily recognized. Repeatability between the observers was good, as measured by the intraclass correlation coefficient (data not shown).

A number of limitations needs to be taken into account when interpreting our results. First, this was a crosssectional study and it cannot be ruled out that our group of ex-smokers quit smoking because they experienced more smoking related symptoms and they may have had different macrophage phenotypes before quitting. In addition, we did not confirm smoking status by laboratory tests which is in line with other cross-sectional studies $[4,5]$ and therefore cannot exclude the possibility that some ex-smokers were still smoking. Second, BAL samples were not available from all subjects in our study due to ethical considerations. As this was not anticipated, it is unlikely that a selection bias for the BAL results was introduced. Nevertheless, a significant difference in antiinflammatory macrophages in BAL was found between smokers and ex-smokers. Further studies are needed to investigate whether the observed differences in CD163 staining on macrophages are also observed when comparing current or ex-smokers without COPD to nonsmokers and whether CD163 expression is a specific feature of COPD. Third, we only focused on the marker CD163 for M2 macrophages, which can result in an oversimplification of our conclusions. Furthermore, it appears that the M2 macrophage population is more heterogeneous than the M1 population [9] and M2 subpopulations were not taken into account in our analysis. Obviously, it is of interest to evaluate whether the use of pro-inflammatory or other anti-inflammatory markers (like arginase or iNOS) can confirm our results and whether associated functional differences can be detected. Currently, there is no general agreement on well defined markers for M1 macrophages.

Fourth, we found that the percentage $\mathrm{CD} 163^{+}$cells is higher in ex-smokers with COPD whereas the number of $\mathrm{CD}_{163^{+}}$cells is higher in current smokers with COPD. In addition, we observed a higher percentage and number of macrophages in BAL from smokers compared to ex-smokers, which likely results from more active recruitment of monocytes from the circulation. Therefore, it is not surprising that smokers have a higher number of $\mathrm{CD}_{163}{ }^{+}$cells in BAL, since they have more macrophages in BAL. We hypothesize that percentages and numbers provide different and complimentary information: percentages better reflect the environment during differentiation, whereas cell numbers result from both recruitment and differentiation. Fifth, several soluble mediators were below the lower limits of detection in sputum and BAL supernatants. Finally, analysis of cytospins using immunocytochemistry is a semi-quantitative measurement and could therefore result in incorrect interpretations. Using e.g. FACS analysis ideally combined with functional analysis of e.g. the phagocytic capacity of the macrophages, could have been more accurate to evaluate the equilibrium between pro- and anti-inflammatory macrophages in our samples. Unfortunately, fresh samples were not available at the time of this research.

How can we explain our results? Macrophages in the periphery of the lung in healthy individuals display mainly anti-inflammatory characteristics that may be involved in suppressing inflammation in this area of the lung. Our study, as well as recent data from others $[19,40]$, suggest that the anti-inflammatory environment may change into a pro-inflammatory environment as COPD develops in smokers. This is in line with the observation that IL-10 levels are lower and GM-CSF and Matrix Metalloproteinase (MMP)-12 levels are higher in sputum and BAL from COPD patients compared to healthy controls $[39,41,42]$. Inflammatory lung diseases, including COPD [43], are characterized by 
increased local production of GM-CSF which may contribute to development of a pro-inflammatory macrophage phenotype in addition to its established effect on neutrophil survival [44]. Macrophages maintain their plasticity even when differentiated into M1 or M2 cells and can switch their phenotype dependent on the presence of appropriate stimuli $[45,46]$. In this study we add to the field that smoking cessation may skew alveolar macrophage heterogeneity towards a more antiinflammatory phenotype as characterized by the M2 marker CD163. Pro-inflammatory macrophages are the predominant phenotype in the central airways, which may be explained by high exposure to pathogens and environmental stimuli compared to macrophages in the peripheral airways. The higher percentage and number of neutrophils in sputum samples are in line with this observation. The predominance of anti-inflammatory macrophages in the periphery of the lung may help to keep this area, which is central to gas exchange, free from excessive inflammation.

Our results suggest that smoking cessation can change macrophage polarization from a pro-inflammatory towards a CD163 expressing anti-inflammatory phenotype, which may decrease inflammation and enhance repair. Our findings of a positive association between a better lung function and more anti-inflammatory M2 macrophages are in line with this. We hypothesize that a shift in macrophage phenotype contributes to further clinical effects of smoking cessation. Therefore, the plasticity of the macrophage phenotype and the possibility to modulate this phenotype may be relevant to the treatment of chronic inflammation, including COPD.

\section{Conclusions}

This study shows that previous smoking cessation may contribute to the anti-inflammatory phenotype of intraluminal macrophages in BAL of ex-smoking COPD patients in vivo. Additional research is needed to further characterize this phenotype and to demonstrate its impact on local inflammation. Furthermore, studies are needed to investigate whether it is restricted to luminal macrophages or is also present in lung tissue. Prospective studies are required to show whether anti-inflammatory treatment contributes to the anti-inflammatory macrophage phenotype in vivo, and whether this contributes to treatment effects on inflammation and clinical outcomes such as lung function decline.

\section{Acknowledgements}

The authors thank the patients for their cooperation in our study. The authors also thank Dr. N.D.L. Savage (Department of Infectious Diseases, LUMC) for his help in generating M1 and M2 macrophages from blood monocytes for FACS assay validation.
The GLUCOLD study group consists of:

University of Groningen and University Medical Center Groningen,

Groningen, The Netherlands, Department of Allergology: H.F. Kauffman and

D. de Reus. Department of Epidemiology: H.M. Boezen, D.F. Jansen, and J.M. Vonk. Department of Pathology: M.D.W. Barentsen, W. Timens, and M.

Zeinstra-Smit. Department of General Practice: A.J. Luteijn, T. van der Molen, and G. ter Veen. Department of Pulmonology: M.M.E. Gosman, N.H.T. ten Hacken, H.A.M. Kerstjens, M.S. van Maaren, D.S. Postma, C.A. Veltman, A. Verbokkem, I. Verhage, and H.K. Vink-Klooster.

Leiden University Medical Center, Leiden, The Netherlands Department of General Practice: H.A. Thiadens. Department of Medical Decision Making: J.B. Snoeck-Stroband and J.K. Sont. Department of Pulmonology: J. GastStrookman, P.S. Hiemstra, K. Janssen, T.S. Lapperre, K.F. Rabe, A. van Schadewijk, J.A. Schrumpf, J. Smit-Bakker, P.J. Sterk, J. Stolk, A.C.J.A. Tiré, H. van der Veen, M.M.E. Wijffels, and L.N.A. Willems.

Academic Medical Center, University of Amsterdam, Amsterdam, The Netherlands Department of Respiratory Medicine: P.J. Sterk.

University of São Paulo, São Paulo, Brazil, T. Mauad.

\section{Author details}

'Department of Pulmonology, Leiden University Medical Center, Leiden, The Netherlands. ${ }^{2}$ Department of Medical Decision Making, Leiden University Medical Center, Leiden, The Netherlands. ${ }^{3}$ Department of Epidemiology, University Medical Center Groningen, University of Groningen, Groningen, The Netherlands. ${ }^{4}$ Department of Pathology, University Medical Center Groningen, University of Groningen, Groningen, The Netherlands. ${ }^{5}$ Department of Pulmonology, University Medical Center Groningen, University of Groningen, Groningen, The Netherlands. ${ }^{6}$ Department of Pulmonology, Academic Medical Center, Amsterdam, The Netherlands.

\section{Authors' contributions}

LIK, TSL, JBS, WT, PJS, DSP and PSH designed the study design and the experiments. DSP and KFR performed the bronchoscopies. JAS, SWW and LIK were responsible for immunocytochemical stainings and cell counting. LIK statistically analyzed the data. LIK and PSH drafted the manuscript. SEB, WT, PJS, KFR and DSP read, critically revised and all authors approved the final manuscript.

\section{Competing interests}

This study was funded by Netherlands Organization for Scientific Research (NWO), Dutch Asthma Foundation (NAF), Stichting Astma Bestrijding (SAB), GlaxoSmithKline (GSK) of the Netherlands, University Medical Center Groningen (UMCG), and Leiden University Medical Center (LUMC).

Received: 3 November 2010 Accepted: 22 March 2011 Published: 22 March 2011

\section{References}

1. Rabe KF, Hurd S, Anzueto A, Barnes PJ, Buist SA, Calverley P, et al: Global strategy for the diagnosis, management, and prevention of chronic obstructive pulmonary disease: GOLD executive summary. Am J Respir Crit Care Med 2007, 176:532-555.

2. Hogg JC, Chu F, Utokaparch S, Woods R, Elliott WM, Buzatu L, et al: The nature of small-airway obstruction in chronic obstructive pulmonary disease. N Engl J Med 2004, 350:2645-2653.

3. Barnes PJ: Immunology of asthma and chronic obstructive pulmonary disease. Nat Rev Immunol 2008, 8:183-192.

4. Rutgers SR, Postma DS, ten Hacken NH, Kauffman HF, van der Mark TW, Koeter GH, et al: Ongoing airway inflammation in patients with COPD who do not currently smoke. Thorax 2000, 55:12-18.

5. Turato G, Di Stefano A, Maestrelli P, Mapp CE, Ruggieri MP, Roggeri A, et al: Effect of smoking cessation on airway inflammation in chronic bronchitis. Am J Respir Crit Care Med 1995, 152:1262-1267.

6. Willemse BWM, ten Hacken NHT, Rutgers B, Lesman-Leegte IGAT, Postma DS, Timens W: Effect of 1-year smoking cessation on airway inflammation in COPD and asymptomatic smokers. Eur Respir J 2005, 26:835-845.

7. Lapperre TS, Postma DS, Gosman MME, Snoeck-Stroband JB, ten Hacken NHT, Hiemstra PS, et al: Relation between duration of smoking cessation and bronchial inflammation in COPD. Thorax 2006, 61:115-121. 
8. Martinez FO, Helming L, Gordon S: Alternative activation of macrophages: an immunologic functional perspective. Annu Rev Immunol 2009, 27:451-483.

9. Mosser DM, Edwards JP: Exploring the full spectrum of macrophage activation. Nat Rev Immunol 2008, 8:958-969.

10. Gordon S, Taylor PR: Monocyte and macrophage heterogeneity. Nat Rev Immunol 2005, 5:953-964

11. Verreck FAW, de Boer T, Langenberg DML, van der Zanden $\mathrm{L}$, Ottenhoff THM: Phenotypic and functional profiling of human proinflammatory type- 1 and anti-inflammatory type-2 macrophages in response to microbial antigens and IFN-\{gamma\}- and CD40L-mediated costimulation. J Leukoc Biol 2006, 79:285-293.

12. Savage NDL, de Boer T, Walburg KV, Joosten SA, van Meijgaarden $\mathrm{K}$, Geluk $A$, et al: Human anti-inflammatory macrophages induce Foxp3 +GITR+CD25+ regulatory T cells, which suppress via membrane-bound TGF\{beta\}-1. J Immunol 2008, 181:2220-2226.

13. Xu W, Roos A, Schlagwein N, Woltman AM, Daha MR, van Kooten C: IL-10producing macrophages preferentially clear early apoptotic cells. Blood 2006, 107:4930-4937.

14. Blumenthal RL, Campbell DE, Hwang P, Dekruyff RH, Frankel LR, Umetsu DT Human alveolar macrophages induce functional inactivation in antigenspecific CD4 T cells. J Allergy Clin Immunol 2001, 107:258-264.

15. Thepen T, van Rooijen N, Kraal G: Alveolar macrophage elimination in vivo is associated with an increase in pulmonary immune response in mice. J Exp Med 1989, 170:499-509.

16. Van den Heuvel MM, Tensen CP, van As JH, van den Berg TK, Fluitsma DM, Dijkstra CD, et al: Regulation of CD 163 on human macrophages: cross-linking of CD163 induces signaling and activation. J Leukoc Biol 1999, 66:858-866.

17. Högger P, Dreier J, Droste A, Buck F, Sorg C: Identification of the integral membrane protein RM3/1 on human monocytes as a glucocorticoidinducible member of the scavenger receptor cysteine-rich family (CD163). J Immunol 1998, 161:1883-1890.

18. Schonkeren D, van der Hoorn ML, Khedoe P, Swings G, van Beelen E, Claas FHJ, et al: Differential distribution and phenotype of decidual macrophages in preecclamptic versus control pregnancies. Am J Pathol 2010, 178:709-717.

19. Hodge S, Hodge G, Ahern J, Jersmann H, Holmes M, Reynolds PN: Smoking alters alveolar macrophage recognition and phagocytic ability: Implications in chronic obstructive pulmonary disease. Am J Respir Cell Mol Biol 2007, 37:748-755.

20. Sica A, Schioppa T, Mantovani A, Allavena P: Tumour-associated macrophages are a distinct M2 polarised population promoting tumour progression: Potential targets of anti-cancer therapy. Eur J Cancer 2006, 42:717-727.

21. Woollard KJ, Geissmann F: Monocytes in atherosclerosis: subsets and functions. Nat Rev Cardiol 2010, 7:77-86.

22. Ricardo SD, van Goor H, Eddy AA: Macrophage diversity in renal injury and repair. J Clin Invest 2008, 118:3522-3530.

23. Lapperre TS, Snoeck-Stroband JB, Gosman MME, Stolk J, Sont JK, Jansen DF, et al: Dissociation of lung function and airway inflammation in chronic obstructive pulmonary disease. Am J Respir Crit Care Med 2004, 170:499-504.

24. Lapperre TS, Willems LNA, Timens W, Rabe KF, Hiemstra PS, Postma DS, et al: Small Airways Dysfunction and Neutrophilic Inflammation in Bronchial Biopsies and BAL in COPD. Chest 2007, 131:53-59.

25. Quanjer PH, Tammeling GJ, Cotes JE, Pedersen OF, Peslin R, Yernault JC: Lung volumes and forced ventilatory flows. Report Working Party Standardization of Lung Function Tests, European Community for Steel and Coal. Official Statement of the European Respiratory Society. Eur Respir J Suppl 1993, 16:5-40.

26. Technical recommendations and guidelines for bronchoalveolar lavage (BAL). Report of the European Society of Pneumology Task Group. Eur Respir J 1989, 2:561-585.

27. Haslam PL, Baughman RP: Report of ERS Task Force: guidelines for measurement of acellular components and standardization of BAL. Eur Respir J 1999, 14:245-248.

28. in't Veen JC, de Gouw HW, Smits HH, Sont JK, Hiemstra PS, Sterk PJ, et al: Repeatability of cellular and soluble markers of inflammation in induced sputum from patients with asthma. Eur Respir J 1996, 9:2441-2447.

29. van Wetering S, van der Linden AC, van Sterkenburg MA, Rabe KF, Schalkwijk J, Hiemstra PS: Regulation of secretory leukocyte proteinase inhibitor (SLPI) production by human bronchial epithelial cells: increase of cell-associated SLPI by neutrophil elastase. J Investig Med 2000, 48:359-366.

30. Frankenberger $M$, Menzel $M$, Betz R, Kaßner $G$, Weber N, Kohlhäufl M, et al: Characterization of a population of small macrophages in induced sputum of patients with chronic obstructive pulmonary disease and healthy volunteers. Clinical \& Experimental Immunology 2004, 138:507-516.

31. Kollert F, Probst C, Muller-Quernheim J, Zissel G, Prasse A: CCL18 production is decreased in alveolar macrophages from cigarette smokers. Inflammation 2009, 32:163-168.

32. Berenson CS, Garlipp MA, Grove LJ, Maloney J, Sethi S: Impaired phagocytosis of nontypeable Haemophilus influenzae by human alveolar macrophages in chronic obstructive pulmonary disease. I Infect Dis 2006, 194:1375-1384.

33. Taylor AE, Finney-Hayward TK, Quint JK, Thomas CMR, Tudhope SJ, Wedzicha JA, et al: Defective macrophage phagocytosis of bacteria in COPD. Eur Respir J 2010, 35:1039-1047.

34. Verreck FAW, de Boer T, Langenberg DML, Hoeve MA, Kramer M, Vaisberg E, et al: Human IL-23-producing type 1 macrophages promote but IL-10-producing type 2 macrophages subvert immunity to (myco) bacteria. Proc Natl Acad Sci USA 2004, 101:4560-4565.

35. Shaykhiev R, Krause A, Salit J, Strulovici-Barel Y, Harvey BG, O'Connor TP, et al: Smoking-dependent reprogramming of alveolar macrophage polarization: Implication for pathogenesis of chronic obstructive pulmonary disease. J Immunol 2009, 183:2867-2883.

36. Hollander C, Sitkauskiene B, Sakalauskas R, Westin U, Janciauskiene SM: Serum and bronchial lavage fluid concentrations of IL-8, SLPI, SCD14 and SICAM-1 in patients with COPD and asthma. Respir Med 2007, 101:1947-1953.

37. Aaron $\mathrm{S}$, Vandemheen $\mathrm{K}$, Ramsay $\mathrm{T}$, Zhang $\mathrm{C}$, Avnur Z, Nikolcheva T, et al: Multi analyte profiling and variability of inflammatory markers in blood and induced sputum in patients with stable COPD. Respiratory Research 2010, 11:41.

38. Stravinskaite K, Sitkauskiene B, Dicpinigaitis PV, Babusyte A, Sakalauskas R: Influence of smoking status on cough reflex sensitivity in subjects with COPD. Lung 2009, 187:37-42.

39. Saha S, Doe C, Mistry V, Siddiqui S, Parker D, Sleeman M, et al: Granulocytemacrophage colony-stimulating factor expression in induced sputum and bronchial mucosa in asthma and COPD. Thorax 2009, 64:671-676.

40. Hodge S, Matthews G, Mukaro V, Ahern J, Shivam A, Hodge G, et al: Cigarette Smoke-induced Changes to Alveolar Macrophage Phenotype and Function is Improved by Treatment with Procysteine. Am J Respir Cell Mol Biol 2010, 2009-04590C.

41. Takanashi S, Hasegawa Y, Kanehira Y, Yamamoto K, Fujimoto K, Satoh K, et al: Interleukin-10 level in sputum is reduced in bronchial asthma, COPD and in smokers. Eur Respir J 1999, 14:309-314.

42. Babusyte A, Stravinskaite K, Jeroch J, Lotvall J, Sakalauskas R, Sitkauskiene B: Patterns of airway inflammation and MMP-12 expression in smokers and ex-smokers with COPD. Respir Res 2007, 8:81.

43. Culpitt SV, Rogers DF, Shah P, De Matos C, Russell REK, Donnelly LE, et al: Impaired inhibition by dexamethasone of cytokine release by alveolar macrophages from patients with chronic obstructive pulmonary disease. Am J Respir Crit Care Med 2003, 167:24-31.

44. Lee E, Lindo T, Jackson N, Meng-Choong L, Reynolds P, Hill A, et al: Reversal of human neutrophil survival by leukotriene B4 receptor blockade and 5-lipoxygenase and 5-lipoxygenase activating protein inhibitors. Am J Respir Crit Care Med 1999, 160:2079-2085.

45. Yanagita M, Kobayashi R, Murakami S: Nicotine can skew the characterization of the macrophage type-1 (MPhi1) phenotype differentiated with granulocyte-macrophage colony-stimulating factor to the MPhi2 phenotype. Biochem Biophys Res Commun 2009, 388:91-95.

46. Stout RD, Jiang C, Matta B, Tietzel I, Watkins SK, Suttles J: Macrophages sequentially change their functional phenotype in response to changes in microenvironmental influences. J Immunol 2005, 175:342-349.

doi:10.1186/1465-9921-12-34

Cite this article as: Kunz et al.: Smoking status and anti-inflammatory macrophages in bronchoalveolar lavage and induced sputum in COPD. Respiratory Research 2011 12:34. 